\title{
Companionship games: a framework for emotionally engaging and empathetic interactive characters
}

Alice Bowman

This is the Author Accepted Manuscript of a conference paper published in Interactive storytelling: 12th International Conference on Interactive Digital Storytelling, ICIDS 2019, Little Cottonwood Canyon, UT, USA, November 19-22, 2019, Proceedings

"The final authenticated version is available online at https://doi.org/10.1007/978-3-030-33894-7_44 


\title{
Companionship Games: A framework for emotionally engaging and empathetic interactive characters
}

\author{
Alice Bowman \\ Abertay University, Bell Street, Dundee, DD1 1HG \\ 1704646@uad.ac.uk
}

\begin{abstract}
. 1 in 2 people are diagnosed with cancer in their lifetime, and for those living with cancer loneliness and isolation are significant problems. This paper discusses the development of a virtual cancer support group, using the support group format to offer a companionship game to breast cancer patients. Seven characters populate this support group, designed as empathetic virtual agents. Interviews and playtests will assess the effectiveness of the design practice developed, and these learnings will be used to create a design framework for emotionally engaging and empathetic interactive characters.
\end{abstract}

Keywords: Interactive Storytelling, Serious Games, Cancer Support, Narrative Design, Character Design

\section{Introduction}

Most of us will be affected by cancer to some extent over the course of our lifetimes. The lifetime risk of receiving a cancer diagnosis for people in the UK born after 1960 has risen to 1 in 2 according to Cancer Research UK [1]. Even those who are not affected directly are likely to see friends or family diagnosed with cancer.

As the likelihood of diagnosis increases and the average lifespan of cancer patients lengthens due to developing treatment options, we must also increase the soft services offered to accommodate the growing population living with cancer. People live with technology, smartphone ownership in the UK has risen to 78\% [2], games and apps have become a part of daily life for many people. Individuals living with cancer are perhaps even more invested in this outlet - a smartphone or tablet could be a lifeline to the outside world to someone who is bedridden, or a doorway into escapism for someone suffering through chemotherapy. With this in mind, I am undertaking the challenge of creating a game to offer additional support to cancer patients.

Specifically, I have chosen to design a virtual cancer support group for breast cancer patients. Loneliness and isolation are significant problems for people living with cancer [3], and when severe can be detrimental to patient prognosis. Accessing a support group can be difficult for a range of reasons, and a virtual cancer support group would provide a supplement or replacement to patients who might not otherwise be able to access these services.

This project is not the first to employ game design practice in an attempt to solve a problem, or otherwise offer a service other than entertainment through the format of games. Serious games are a genre made up of games that aim to engage their players beyond fun or entertainment [6], 
and a sub-genre of serious games deal with topics relating to healthcare and wellbeing. Some of these games have specifically addressed issues around cancer - That Dragon Cancer [7] is a biographical game made by a developer to depict his young child's experience of terminal cancer and the impact on his family. Beyond this, other games such as Re-Mission [8] and The Cancer Game [9] allow players to play out actions of fighting cancer and destroying cancerous cells - these games can be cathartic for players of any age, and can be used to help younger patients frame the often traumatising treatment in positive terms. The area of companionship games is less explored, but the greater context of serious games and the topic of cancer in gaming provides some insight in the form of critical play with which to prime this research.

\section{Research Aims}

Previous studies and literature indicate that players can develop meaningful attachments to game characters and games themselves [4]. The driving question behind this research is how can characters be designed to foster these emotions in the context of a serious game? Previous papers have examined the potential for emotional authenticity in virtual agents [5]. This research seeks to test the extent to which emotional authenticity and feelings of empathy and companionship can be generated using characters designed to be empathetic virtual agents.

The prototype developed for this project aims to use a fictional simulation of a support group to provide companionship to breast cancer patients and alleviate loneliness. For patients who are unable to access support groups, the app will offer an alternative that can provide some of the emotional support that patients can derive from support groups. For patients who can access support groups, the app might be supplementarily allowing them to access emotional support when their group is not running, or might also serve as a transitional tool for patients who are tentative about accessing a group.

Ultimately this research will enable the development of a design framework identifying the core qualities of the empathetic virtual agents required for companionship games, as well as defining what a companionship game is. This style of game might be used in any scenario where a user group could benefit from the emotional support that empathetic virtual agents can provide. Additionally, it could be used to educate users about the emotions and reality of living with cancer, and might provide empathy training.

\section{Methodology and Process}

A preliminary literature review of comparable work and relevant research was carried out at the start of this work to prime the early stages of planning this research. This literature review acts as a foundation for the research being undertaken and has also been used to build a case as to the value of the research and reasoning for the hypothesis of players benefiting from emotional attachment with virtual characters.

A thematic analysis of patient forums and online communities of people living with cancer was carried out. This was the first step in developing an awareness of the experiences of cancer patients, and an understanding of what it can be like to live with cancer. This knowledge has been important to the project as it has facilitated the accurate representation of patients and 
their experiences, and was a good preparatory step prior to carrying out further research with patients.

Following this early research, I began working on designs for a virtual support group prototype, designing the game flow and some potential characters. This design work was done in parallel to other continued streams of research and allowed me to explore the project through practice and prototyping. This practice as research has been the core of the project and has continued throughout the development of this work, drawing on my background in game design and working within a framework of design literature that discusses emotional bonds between players and characters.

A series of 1-1 interviews with breast cancer patients are currently being carried out. These interviews are semi-structured, focusing on topics such as loneliness, isolation and emotional wellbeing. These interviews are hosted by Dundee's Maggie's Centre, the local branch of a UKwide cancer charity. These interviews are being used to inform the narrative and character design, enriching the stories with realistic detail and ensuring that they are accurate representations of the patient experience. The narrative and characters do not directly depict any of the individuals interviewed or their experiences, but the qualitative data gathered has informed the design practice.

A co-design session with breast cancer patients will then be used to examine the prototype and gather feedback that can be used to iterate on the game's design and hone the narrative and characters. Patients will be asked to list the topics they would be likely to discuss at a support group, and will be asked about who they would most want to discuss each topic with. The character designs will be broken into components (ie. individual personality traits, age, background, appearance) so participants can create their own variations on characters. This session will be used to explore player preference in identifying companions and to better understand what makes a player recognise a fictional character as an empathetic confidant.

Through this research, the prototype will be iterated on to account for design learnings and insights that the character design framework provides as it develops. The final prototype of the game will be playtested at breast cancer support groups in brief half-hour play sessions to capture initial player reactions. A small sample of breast cancer patients will be asked to participate in an extended study of engaging with the game over a longer period of time and playing the content in full. This extended playtest will be followed by individual interviews to gather feedback on the player's experience and feelings towards the game and characters.

\section{Prototype}

The prototype is currently in development, and this practice is a key component of the research being undertaken. It is being made in Unity using the Fungus plug-in, which gives developers a series of flow-chart based scripts to direct dialogue and branching narrative. Seven characters have been designed as fictional breast cancer patients to populate the support group. The first iteration of these character designs has been based on a combination of early research and practice-based experimentation ideating through multiple concepts. These character designs 
will evolve as the research progresses and provides more material to inform the design. At present, the characters have been written to encourage a close and empathetic bond with the player, and their backgrounds have been designed so as to represent an array of different types of patient.

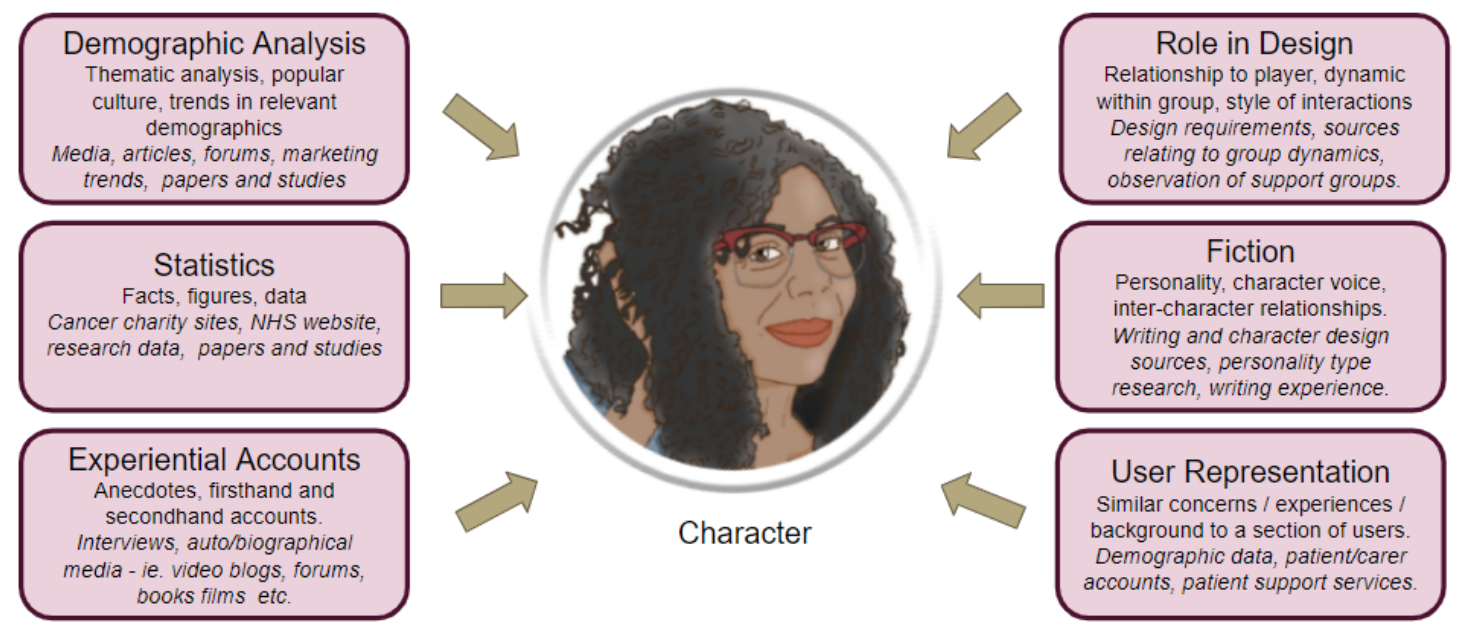

The above image shows the working framework for designing empathetic virtual agents in companionship games. At present this framework is built off of practice-based research, a literature review of relevant work and the other types of research undertaken so far, as discussed in the above section. This framework will likely evolve as the research continues, the current iteration of the character designs have been developed using the framework, and their dialogue, character arcs and interactions with the player all reflect the impact of this framework. These aspects of the prototype can all be iterated to accommodate changes to the framework based on new findings.

\section{Next Steps}

Patient interviews are still ongoing, and they will be followed by the co-design session, and later playtesting of the prototype. The prototype is currently in development, with the first iteration almost complete, and will continue to evolve to reflect the new learnings uncovered by the ongoing research. In presenting this research to the ICIDS Doctoral Consortium I would like to gather feedback specifically on the research methods employed and the development of the character design framework. These two components of the project both have a significant impact on the validity of the research being undertaken, and it will be crucial in the dissemination of this research that the reasoning behind the methods and the output of the framework can be explained clearly and compellingly.

\section{References}

[1] Cancer Research UK. (Online). 'Cancer Statistics for the UK'. Available at: https://www.cancerresearchuk.org/health -professional/cancer -statistics -for-the-uk (Accessed: 1 August 2019)

[2] Ofcom (2018) Ofcom Communications Market Report 2018 Available at: https://www.ofcom.org.uk/ data/assets/pdf file/0022/117256/CMR-2018-narrative-report.pdf (Accessed: 1 August 2019) 
[3] Macmillan (2013) Isolation among cancer patients. Available at:

https://www.macmillan.org.uk/documents/aboutus/newsroom/isolated cancer patients media $r$ eport.pdf (Accessed: 1 August 2019)

[4] Mallon, B., \& Lynch, R. (2014). Stimulating Psychological Attachments in Narrative Games:

Engaging Players With Game Characters. In Simulation \& Gaming.

[5] Turkle, S. (2007). Authenticity in the age of digital companions. In Interaction Studies - Social Behaviour and Communication in Biological and Artificial Systems.

[6] Chen, S. and Michael, D. (2005) Serious Games: Games That Educate, Train and Inform.

Boston: Course Technology PTR.

[7] Numinous Games. That Dragon Cancer. (2016) Unity

[8] Realtime Associates. Re-Mission. (2006) Hopelab

[9] Oda, Y and Kristual, D. The Cancer Game. (2014) Albright College 